\title{
Quantitative Evaluation of the Amount of Delayed Myocardial Enhancement as a Predictor of Systolic Dysfunction
}

\author{
J.E. Shriki ${ }^{1}$, K. Surti ${ }^{1}$, A. Farvid ${ }^{2}$, J.S. Shinbane ${ }^{2}$ and P.M. Colletti ${ }^{1,2, *}$ \\ Departments of Radiology ${ }^{I}$ and Medicine ${ }^{2}$, Keck School of Medicine, University of Southern, California, USA
}

\begin{abstract}
: 30 patients with delayed contrast enhancement in patterns suggestive of myocardial infarctions were reviewed. Infarct mass was quantitatively measured using short axis images obtained in the delayed phase of gadopentetate administration. Left ventricular mass and ejection fraction were measured using short axis, steady state free precession images. A relationship is drawn between increased mass of infarction and decreased left ventricular ejection fraction. For each gram of infarct, there is a $0.5 \%$ reduction in ejection fraction $(\mathrm{EF}=50-(0.48 \mathrm{x}$ gm infarcted myocardium); $\left.r^{2}=0.49\right)$. For each $\%$ increase of infarcted myocardium, there is a $0.67 \%$ reduction in ejection fraction $(E F=50-(0.67 \mathrm{x}$ percent of infarcted myocardium); $\left.r^{2}=0.39\right)$. Left ventricular ejection fraction correlates inversely with the mass of myocardium with delayed enhancement on cardiac MRI.
\end{abstract}

\section{INTRODUCTION}

Delayed contrast enhancement MRI (DCE-MRI) has gained widespread acceptance as an accurate means to delineate myocardial fibrosis. Myocardial fibrosis patterns consistent with myocardial infarction are derived from patterns with subendocardial to progressive transmural involvement in an anatomic distribution consistent with coronary anatomy. As cardiac MRI has become more commonly utilized, further applications of this technique have developed including evaluation of other cardiomyopathies. Although several studies have showed the validity of DCE for measuring infarct size and predicting nonviability, further work is needed to establish the significance of quantitative assessment of infarct size using DCE-MRI.

The ability to predict the left ventricular ejection fraction based on infarct volume size adds a new diagnostic dimension to delayed contrast enhanced MRI. This retrospective study is performed to assess the quantitative relationship between infarct size as defined by DCE and the degree of decrease in ejection fraction in order to define whether a reliable prediction in the degree of decreased systolic function can be made based on quantification of the size of the infarct as shown by DCE-MRI.

\section{METHODS}

\section{Patient Population}

Thirty consecutive patients from a large, county hospital were included in the analysis. Inclusion criteria consisted of presence of an infarction in the left ventricle, visible on DCE-MRI and completion of the MRI study. Patients were included in the study only if the morphology of areas of

*Address correspondence to this author at the MRI Fellowship Chief of MRI, LAC+USC Imaging Science Center, 1744 Zonal Ave. Los Angeles, CA 90033, USA; Tel: 323-221-2744; Fax: 323-221-2982;

E-mail: colletti@usc.edu delayed contrast enhancement conformed to the expected shape and distribution of an infarction (e.g. subendocardial progressive to transmural delayed enhancement in the expected vascular distributions of the coronary arteries [1]. Twenty four of the patients were male, the remaining 6 were female. The mean age of the population was 54 years, with a range from 44 to 68 .

\section{MRI TECHNIQUE}

Studies were performed between June 21st, 2006 and April 3rd, 2007. All studies were performed on a GE Signa 1.5T field strength MRI unit. Computation of systolic function was based on short axis, balanced steady state free precession sequences (Fast Imaging Employing Steady State Acquisition (FIESTA)). Contiguous $8 \mathrm{~mm}$ short axis images were obtained during breath-holding and using segmental $\mathrm{k}$-space acquisition gated to the cardiac cycle. Ten minutes after the intravenous administration of Magnevist (Bayer, Wayne, NJ), $0.2 \mathrm{mmol} / \mathrm{kg}$, and delayed contrast enhanced contiguous $8 \mathrm{~mm}$ short axis images were obtained during breath-holding using a gradient-echo inversion recovery sequence with inversion time optimized to null normal myocardial signal. Processing was performed on an ADW workstation (General Electric, Milwaukee, WI) using Mass Analysis Plus (Medis, Leiden, The Netherlands). Regions of interest were manually drawn around the left ventricle at end systole and end diastole on the functional images (Fig. 1) and around the areas of delayed myocardial enhancement (Fig. 2). These regions were used to generate quantitative functional data including assessment of ejection fraction (LVEF), end systolic and end diastolic volumes, stroke volumes, assessment of left ventricular (LV) mass and myocardial infarct volume. Comparisons were made between the size of infarction and the left ventricular ejection fraction.

\section{RESULTS}

Our analysis shows that there is a predictable, linear relationship between infarct size and degree of systolic 
dysfunction (Table 1). Two parameters specifically were correlated to assess the relationship between infarct size and depressed systolic function.

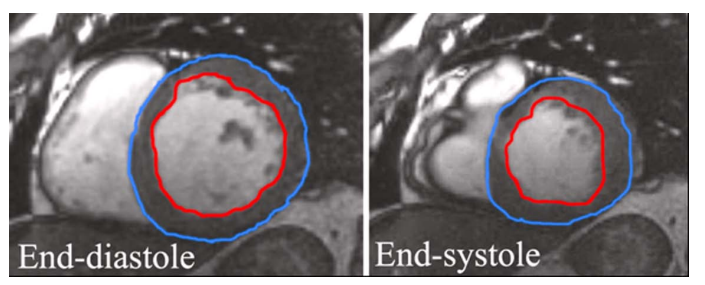

Fig. (1). Regions drawn at end-systole and end-diastole. DCEMRI sequences were obtained at a time delay of ten to fifteen minutes and using an inversion time (TI) of $200 \mathrm{~ms}$. Quantification of infarct size was processed on an ADW workstation as well, based on short axis images.

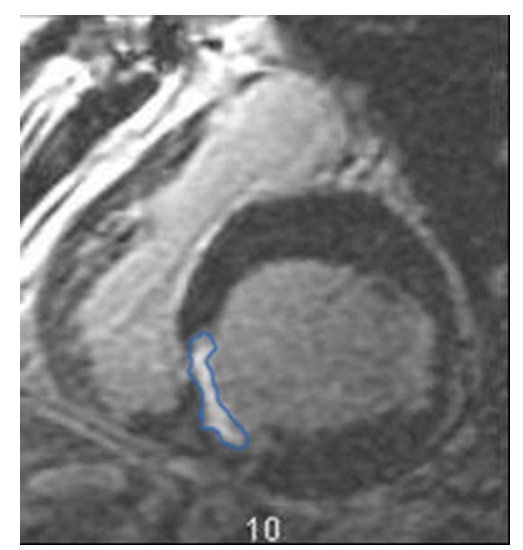

Fig. (2). Region drawn around area of infarction.

First, we drew a relationship between infarct mass and ejection fraction. Infarct mass was shown to correlate with ejection fraction according to the following relationship:

Ejection Fraction $(\%)=-0.48 \times$ (grams of infracted myocardium) $+50 \mathrm{r}^{2}=0.4941$.

In other words, from a baseline ejection fraction of 50\%, each gram of myocardium infracted would be expected to decrease the ejection fraction by roughly $0.5 \%$. (Fig. 3).

Second, we drew a relationship between infarct percentage and ejection fraction. Infarct percentage was calculated by again deriving an infarct mass as described above and dividing the amount of infarcted myocardium by the total left ventricular mass. Infarct percentage was shown to correlate with ejection fraction according to the following relationship:

Ejection Fraction $(\%)=-0.67 \times(\%$ mass of infarcted myocardium) $+49 \mathrm{r}^{2}=0.3919$.

In other words, from a baseline ejection fraction of roughly $50 \%$, each incremental increase in percentage of myocardium infarcted would be expected to decrease the ejection fraction by $0.66 \%$. (Fig. 4).

\section{DISCUSSION}

Ejection fraction is an important factor in assessment of cardiovascular morbidity and mortality [1-5]. There is indeed, an important relationship between infarct size, decreased systolic function, and subsequent cardiovascular morbidity and mortality $[6,7]$.
Table 1. LVEF, Infarct Mass, and Infarct Percent

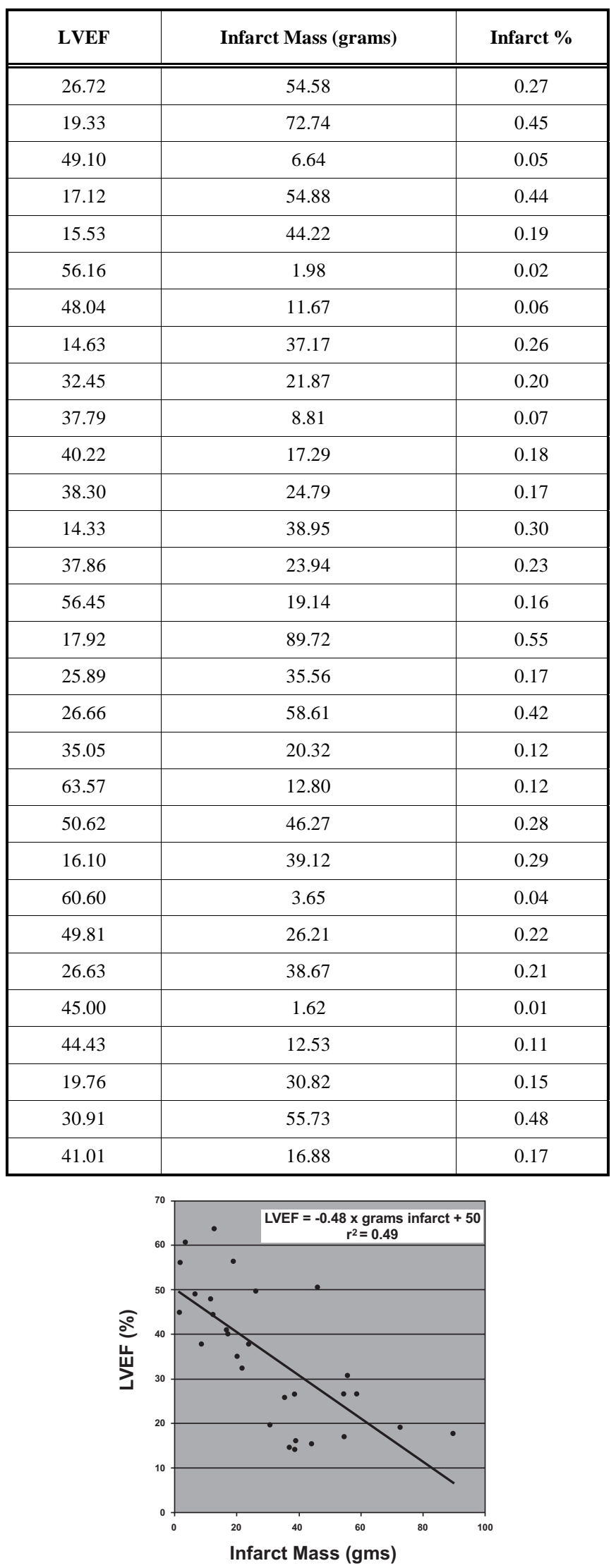

Fig. (3). Relationship of left ventricular ejection fraction (LVEF) to infarct volume. $\mathrm{LVEF}=-0.48 \mathrm{x}$ grams infracted myocardium $+50, r^{2}=0.4941$. 


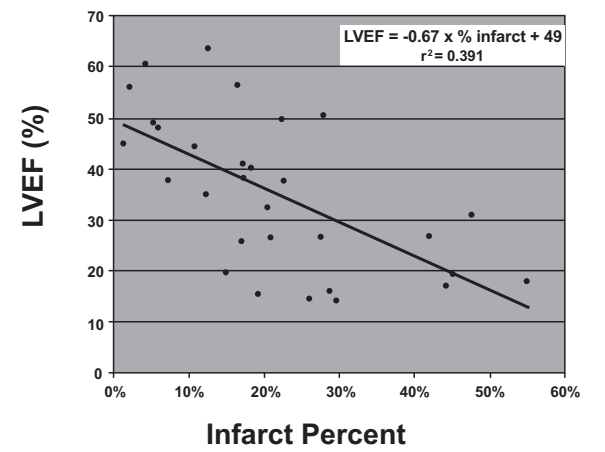

Fig. (4). Relationship of LVEF to infarct percent. $L V E F=-0.67$ $\mathrm{x} \%$ infarct mass $+49, \mathrm{r}^{2}=0.3919$.

Previously, infarct size has been measured noninvasively by several other imaging modalities and correlated with worsened systolic function. This has been employed most widely with scintigraphy [8-12], but has also been shown with estimations of infarct size based on serum enzyme evaluation. [13-15] Efforts to evaluate infarct size by studies evaluating motion such as echocardiography usually overestimate infarct size, since non-transmural infarctions affecting the sub-endocardium will cause disproportionately larger areas of wall motion abnormalities [16, 17].

MRI more recently been validated in both animal models and in humans as demonstrating excellent correlation with pathologic size of areas of infarction. The superior spatial resolution of MRI allows better evaluation of overall size of infarction and can also enable delineation of degree of wall thickness involvement in infarction. [18-20]

Other studies have shown links between infarct size and wall motion abnormalities. Marholdt et al. showed that wall motion abnormalities ensue when more than $20 \%$ of wall thickness is infarcted in a given segment. [21] Beek et al. have shown that the likelihood of recovery of function diminishes when $>50 \%$ of wall thickness is infarcted [22]. These investigations have shown the utility of evaluating the degree of transmurality of infarctions, which is best demonstrated by MRI [23].

This study further supports the link between infarct size and ejection fraction. Also, we further show that in addition to proportionality, there is a quantifiable, direct relationship between the size of the infarct and the degree of systolic dysfunction. This is the largest study, to date, to show this linkage. To our knowledge, this is also the first study to quantify the predictability of systolic dysfunction from DCE-MRI.

There are several implications from this data that this analysis does not directly address. First, it follows syllogistically that if infarct size is correlated with worsened systolic function and depressed ejection fraction contributes to increase cardiac morbidity and mortality, then the amount of infarction measured by DCE-MRI may be an important indicator of subsequent morbidity and mortality. At our institution, data collection is currently underway to better evaluate this relationship.

Additionally, several outliers in the data sets are present. These consist of patients who either had smaller infarct size but more severe systolic dysfunction or patients who had larger infarcts but relatively preserved function.
Although there was heterogeneity in the patient population studied herein, it would be helpful to identify patients who may lie in the former category, with relatively severe systolic dysfunction for the degree of infarct size. This patient population would be important to identify for two reasons. First, we would posit that in patients with relatively small infarct size but severe systolic dysfunction, there may be a greater benefit for subsequent revascularization. A follow-up study showing a function-DCE mismatch might be useful in patients who should be more aggressively considered for revascularization. Second, identifying patients with a systolic function-DCE mismatch might be related to a superimposed cardiomyopathy.

Relatively fewer patients were identified with relatively with larger infarct size had preserved systolic function. The finding of few patients in this latter category further underscores that worsened DCE is associated with more deteriorated function. Further study could be directed at optimization of function in the setting of large infarctions. A benchmark for the utility of treatment options would be pushing the patient rightward in the direction of the abscissa on the graphs presented, e.g. achieving improvements in systolic function in the face of larger infarctions.

Finally, another implication of the data presented is in the better reliability of the relationship between infarct size and ejection fraction compared with infarct percentage and ejection fraction. Based on this relatively small study sample size, it is likely that infarct size is a better predictor of functional impairment than infarct percentage. This makes sense since in calculating infarct percentage; the denominator used is left ventricular mass. Although increased left ventricular mass and remodeling may be useful in order to preserve systolic function, beyond a certain point, enlargement of the left ventricle becomes dysfunction. In patients with an enlarged left ventricle, it is suspected that an infarction might result in an even greater impairment in contractility. This contention is born out in the fact that overall infarct size, rather than infarct percentage is a more reliable predictor of left ventricular dysfunction.

We acknowledge several limitations in this study. Specifically, there was some heterogeneity in the patient populations. Many of the patients were imaged at least one month after the date of infarction. However, for many patients, the date of infarction was difficult to ascertain, by history. Hombach et al. [23] have shown that functional recovery is maximized at one month after infarction.

An additional limitation of this study is the possible overlap in patients who have ischemic and other, superimposed etiologies for cardiomyopathy. All of the studies included in this analysis were screened by an experienced cardiac MRI reader to ensure that the areas of delayed enhancement had morphology consistent with infarction. On the other hand, however, it is impossible to ensure that the patients included do not have a superimposed non-ischemic cardiomyopathy.

\section{CONCLUSIONS}

Percent myocardium with delayed enhancement correlates inversely with ejection fraction in an incremental manner, where from a baseline ejection fraction of $50 \%$, 
each gram of myocardium infracted is correlated with a decrease in left ventricular ejection fraction of $0.5 \%$.

\section{REFERENCES}

[1] van den Broek SA, van Veldhuisen DJ, de Graeff PA, Landsman ML, Hillege H, Lie KI. Comparison between New York Heart Association classification and peak oxygen consumption in the assessment of functional status and prognosis in patients with mild to moderate chronic congestive heart failure secondary to either ischemic or idiopathic dilated cardiomyopathy. Am J Cardiol 1992; 70: 359-63.

[2] Likoff MJ, Chandler SL, Kay HR. Clinical determinants of mortality in chronic congestive heart failure secondary to idiopathic dilated or to ischemic cardiomyopathy. Am J Cardiol 1987; 59: 634-8.

[3] Juillière Y, Barbier G, Feldmann L, Grentzinger A, Danchin N, Cherrier F. Additional predictive value of both left and right ventricular ejection fractions on long-term survival in idiopathic dilated cardiomyopathy. Eur Heart J 1997; 18: 276-80.

[4] Hallstrom A, Pratt CM, Greene HL, et al. Relations between heart failure, ejection fraction, arrhythmia suppression and mortality: analysis of the Cardiac Arrhythmia Suppression Trial. J Am Coll Cardiol 1995; 25: 1250-7.

[5] Pernenkil R, Vinson JM, Shah AS, Beckham V, Wittenberg C, Rich MW. Course and prognosis in patients $\geq 70$ years of age with congestive heart failure and normal versus abnormal left ventricular ejection fraction. Am J Cardiol1997; 79: 216-9.

[6] The Multicenter Post infarction Group. Risk stratification and survival after myocardial infarction. N Engl J Med 1983; 309: 3316.

[7] White HD, Norris RM, Brown MA, Brandt PW, Whitlock RM, Wild CJ. Left ventricular end-systolic volume as the major determinant of survival after recovery from myocardial infarction. Circulation 1987; 76: 44-51.

[8] Van de Werf F. Discrepancies between the effects of coronary reperfusion on survival and left ventricular function. Lancet 1989; 1: $1367-9$.

[9] Silverman KJ, Becker LC, Bulkley BH, et al. Value of early thallium-201 scintigraphy for predicting mortality in patients with acute myocardial infarction. Circulation 1980; 61: 996-1003.

[10] Becker LC, Silverman KJ, Bulkley BH, Kallman CH, Mellits ED, Weisfeldt M. Comparison of early thallium-201 scintigraphy and gated blood pool imaging for predicting mortality in patients with acute myocardial infarction. Circulation 1983; 67: 1272-82.
[11] Chareonthaitawee P, Christian TF, Hirose K, Gibbons RJ, Rumberger JA. Relations of initial infarct size to extent of left ventricular remodeling in the year after acute myocardial infarction. J Am Coll Cardiol 1995; 25: 567-73.

[12] Botvinick EH, Perez-Gonzalez JF, Dunn R, Ports T, Chatterjee K, Parmley W. Late prognostic value of scintigraphic parameters of acute myocardial infarction in complicated myocardial infarction without heart failure. Am J Cardiol 1983; 51: 1045-51.

[13] Hackel DB, Reimer KA, Ideker RE, et al. Comparison of enzymatic and anatomic estimates of myocardial infarct size in man. Circulation 1984; 70: 824-35.

[14] Smith JL, Ambos HD, Gold HK, et al. Enzymatic estimation of myocardial infarct size when early creatine kinase values are not available. Am J Cardiol 1983; 51: 1294-300.

[15] Kim RJ, Fieno DS, Parrish TB, et al. Relationship of MRI delayedcontrast enhancement to irreversible injury, infarct age, and contractile function. Circulation 1999; 100: 1992-2002.

[16] Force T, Kemper A, Perkins L, Gilfoil M, Cohen C, Parisi AF. Overestimation of infarct size by quantitative two-dimensional echocardiography: the role of tethering and of analytic procedures. Circulation 1986; 73: 1360-8

[17] Homans DC, Pavek T, Laxson DD, Bache RJ. Recovery of transmural and subepicardial wall thickening after subendocardial infarction. J Am Coll Cardiol 1994; 24: 1109-16.

[18] Fieno DS, Kim RJ, Chen EL, Lomasney JW, Klocke FJ, Judd RM. Contrast-enhanced magnetic resonance imaging of myocardium at risk: distinction between reversible and irreversible injury throughout infarct healing. J Am Coll Cardiol 2000; 36: 1985-91.

[19] Simonetti OP, Kim RJ, Fieno DS, et al. An improved MR imaging technique for the visualization of myocardial infarction. Radiology 2001; 218: 215-23.

[20] Mahrholdt H, Wagner A, Holly TA, et al. Reproducibility of chronic infarct size measurement by contrast-enhanced magnetic resonance imaging. Circulation 2002; 106: 2322-7.

[21] Mahrholdt H, Wagner A, Parker M, et al. Relationship of contractile function to transmural extent of infarction in patients with chronic coronary artery disease. J Am Coll Cardiol 2003; 42: 50512.

[22] Beek AM, Kuhl HP, Bondarenko O, et al. Delayed contrastenhanced magnetic resonance imaging for the prediction of regional functional improvement after acute myocardial infarction. J Am Coll Cardiol 2003; 42: 895-901.

[23] Hombach V, Grebe O, Merkle N, et al. Sequelae of acute myocardial infarction regarding cardiac structure and function and their prognostic significance as assessed by magnetic resonance imaging. Eur Heart J 2005; 26: 549-57. 